\section{The parallel-less Poggendorff: Virtual contours put the illusion down but not out*}

\author{
MARC B. GOLDSTEIN† and DANIEL J. WEINTRAUB \\ University of Michigan, Ann Arbor, Michigan 48104
}

Four versions of Poggendorff displays were compared: (1) the conventional display of a transversal interrupted by parallel lines, (2) a transversals-only display, (3) a virtual-contour display where the parallels were suggested (but not drawn) via "good continuation" and "closure," and (4) the profile display, a degraded version of the virtual-contour display that retained many of its borders but did not suggest parallels. Each display was drawn with transversal tilted at 45 and $51 \mathrm{deg}$ from vertical. All displays produced significant illusions at both tilts. By comparison with the illusion magnitudes of the conventional display, the effects for the other displays were small. The outcome supports the existence of two salient illusion-producing factors: the necessity for actual contours to form the subtended angle and assimilation of the transversal toward O's horizontal.

Farné (1970), in a note to Cumming (1968), indicated that a modified Poggendorff display, containing remote contours instead of the usual pair of parallel lines, is capable of inducing an illusion. The remote contours, by means of closure and good continuation, suggest the presence of a rectangle between the transversal sections (Fig. 1). Farné also referred to a study by Wundt (1893), who used a degraded display, the transversal segments only, and found that the resulting illusion was small by comparison with the one induced by a conventional Poggendorff figure.

The case of the missing parallels is important because certain theoretical positions are based upon the presence of intersecting contours: Chiang's (1968) hypothesis of retinal blurring of the intersection; the Blakemore, Carpenter, and Georgeson (1970) neurophysiological model backed by psychophysical data; and the receptive-field evidence from single-cell recordings by Burns and Pritchard (1971). These investigators believe that intersecting contours are misrepresented, at some level in the nervous system, such that acute angles are experienced as larger than their objective size warrants. Indeed, this misperception can readily explain the

*Supported by a United States Public Health Service Predoctoral traineeship under Health Service Predoctoral traineeship under Psychology, University of Michigan, awarded to M.B.G., and by a United States Public Health Service Research Scientist Development Award (K2-MH-35,253) to D.J.W. Research funds were provided by D.J.W. Research funds were provided by National Science Foundation
Grant GB 8181 . We thank Lillian Tong for serving as the $\mathbf{E}$.

tAddress: Human Performance Center, Perry Building, 330 Packard Road, Perry Building, 330 Packard Road,
University of Michigan, Ann Arbor, Michigan 48104. here. The important point is that
Poggendorff illusion, i.e., why Os judge a correctly aligned upper transversal segment as being too high.

Another faction presents evidence that it is obtuse angles, not acute angles, that are critical. Green and Hoyle (1964) and Restle (1969) demonstrated a small reversal in the direction of the illusion when the segments of the parallels forming the obtuse angles were deleted. Weintraub and Krantz (1971) parametrically reduced the reflectance contrast of the segment of the parallel forming the obtuse angle and found that, as the line segment was faded out, the illusion was gradually reduced to zero. In addition, they showed that deleting that portion of the parallel which formed the acute angle resulted in only a moderate reduction in illusion magnitude.

Whether the critical feature is the acute or obtuse angle is not at issue either angle-misperception hypothesis relies upon the actual presence of contours forming the angle; the conventional Poggendorff display contains both acute and obtuse angles.
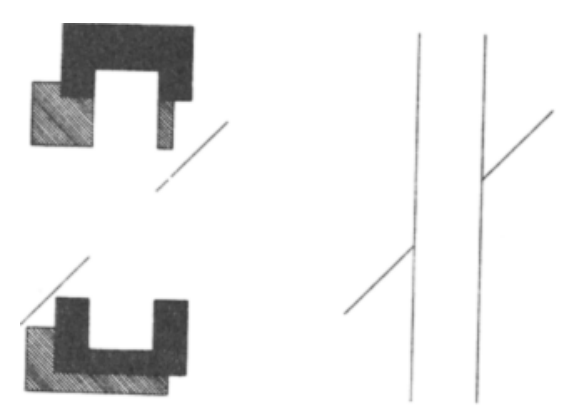

Fig. 1. From left to right, Farné (F) figure, conventional (C) Poggendorff, transversal-only (T) figure, and the profile (P) figure. Cross-hatched regions represent gray. The upper transversal segment was movable by $O$.
Weintraub and Krantz (1971; see also Weintraub \& Virsu, 1971) have uncovered a second factor relevant to the Poggendorff illusion. Descriptively, an $O$ appears to reduce the angular disparity between a line segment and the nearer (in degrees of tilt) cardinal viewing axis. That is, a tilted line will appear as either more horizontal or vertical relative to $O$ 's visual axes. The tilt-assimilation hypothesis predicts that a line tilted more than $45 \mathrm{deg}$ from vertical will appear more horizontal than it actually is. A line with a 45-deg tilt, however, being equidistant from both viewing axes, will not be affected by assimilation processes.

In the absence of any other factors, changing the tilt of the transversal from 45 to 51 deg should produce an increase in the Poggendorff illusion. However, as discussed above, there is considerable evidence to suggest that, in addition to any tilt-assimilation process, an angle misestimation factor is operating on angles defined by the figure's contours. How these two error mechanisms interact is partially illuminated by data from Burmester (1896) and Weintraub and Krantz (1971) which show that, for parallels oriented vertically, the illusion is equal to $(k W / \tan A)$, where $k$ is a constant, $W$ is the width between parallels, and $A$ is the acute angle between transversal and parallel. Thus, the illusion decreases as the acute angle increases above $45 \mathrm{deg}$, even though the tilt-assimilation factor is acting to offset the declining effect of angle misperception. One must conclude that, when both factors are at work, the angle-misperception effect is stronger.

What can be predicted about Poggendorff displays without parallels? With no explicit obtuse (acute?) angles present, only the tilt-assimilation factor should be operating. The transversal-only figure used by Wundt (1893) should give: (1) no illusion when the transversal is oriented at $45 \mathrm{deg}$, and (2) a slight 
Poggendorff effect when the transversal is positioned nearer to horizontal. The Farné (1970) figure in the iournal was measured, and the transversal is tilted $51 \mathrm{deg}$ from vertical. Can it be that the illusion derived from this figure is the result of tilt assimilation and not angle misperception? If such is the case, reducing the transversal tilt to $45 \mathrm{deg}$ should banish the illusion. On the other hand, a conventional Poggendorff display containing parallels and a 45-deg transversal should induce a sizable illusion, since angle misperception is still occurring.

\section{EXPERIMENT 1 Method}

Stimuli. Two versions of Farné's (F) figure, one transversal-only (T) figure similar to Wundt's, and one conventional Poggendorff (C) figure, were drawn on $23 \times 30.5 \mathrm{~cm}$ white paper. These drawings did not include the upper transversal segment. The finished displays are illustrated in Fig. 1. All figures were drawn with the transversal tilt-the acute angle between transversal and vertical-at $45 \mathrm{deg}$, except for the second Farne figure, which had a tilt of 51 deg to match the figure depicted in his article (Farné, 1970). The distance between the parallels was $40 \mathrm{~mm}$; this separation was maintained in all stimuli. The upper transversal was inked onto the underside of a transparent $22.8 \times 40.6 \mathrm{~cm}$ plastic overlay. Two overlays were prepared, one with a transversal tilted at $51 \mathrm{deg}$ to match the F-51-deg figure and the other with a 45-deg tilt to match the remaining displays. India ink (33\% reflectance) and gray paint (44\% reflectance) (gray areas represented by cross hatching in Fig. 1) were used in preparing the stimuli. The reflectance of the white paper was $85 \%$. All reflectance values were taken with the matte-surface transparent overlay in place, as viewed by the $O$. The matte finish eliminated surface reflections but also reduced the figure-ground contrast of the stimuli. Hluminance on the displays was $40 \mathrm{fc}$. The drawing-overlay combination was displayed horizontally on a wooden board that held the underlay in place while allowing the plastic overlay to move freely up or down. The $O$ stood at the edge of a table and peered down at a display through a viewing mask with narrow eye slits. This procedure minimized head movements and maintained eye-to-screen distance at $54 \mathrm{~cm}$.

Design. Forty-three students from the paid $S$ pool at the University of Michigan served in the experiment. The Os were tested individually and made one judgment per figure.
Table 1

Means and Standard Errors of the Mean (in Millmeters) for Each Display

\begin{tabular}{cccccc}
\hline $\begin{array}{c}\text { Trans- } \\
\text { versal } \\
\begin{array}{c}\text { Tilt } \\
\text { (in Deg) }\end{array}\end{array}$ & Farne & $\begin{array}{c}\text { Conven- } \\
\text { tional }\end{array}$ & $\begin{array}{c}\text { Trans- } \\
\text { versal }\end{array}$ & Profile & Imaginary \\
\cline { 2 - 6 } & 1.92 & 7.05 & .46 & 1.56 & -.15 \\
& $(.44)$ & $(.74)$ & $(.37)$ & $(.52)$ & $(.56)$ \\
51 & 3.10 & 5.49 & 1.90 & 2.06 & 1.68 \\
& $(.39)$ & $(.49)$ & $(.38)$ & $(.45)$ & $(.52)$ \\
\hline
\end{tabular}

Note-Italicized values are the data from Experiment 1. Standard errors of the mean are enclosed in parentheses.

Participants were instructed to position the plastic overlay so that the upper and lower transversal segments appeared collinear; however, only vertical movements (up or down) of the overlay were permitted. The starting position of the upper transversal on each trial was either obviously too high or obviously too low, randomized across figures. Vertical errors in alignment were measured with positive values assigned to distances below the true point of collinearity. The individual stimulus displays were presented alternately on two tables, separated by a partition, which permitted $O$ to work on one display while $\mathrm{E}$ measured the other. No feedback was given during the experimental session. Order of presentation of the four stimuli was randomized anew for each $O$.

\section{Results}

The mean amounts of illusion for each display and the associated standard errors of the mean are shown in italics in Table 1. Every display gave a significantly different level of illusion from any other display $(\alpha=.05)$, although the illusory effects of the transversal-only figure were not reliably different from zero $(\alpha=.05)$.

Discussion

Inspection of these data suggest that Farné's (1970) claim concerning a virtual-contour Poggendorff is supported, much to the first author's delight and the second author's dismay. Reducing the tilt of the transversal did not eliminate the illusion. The illusion, however, is much less potent than the one produced by the conventional figure. No support was garnered for Wundt's contention that the $T$ figure gives rise to an illusion.

Upon further reflection, it appeared that several hypotheses about the source of the virtual-contour Poggendorff were tenable. It may arise, as Farné claimed, from perceiving the rectangle suggested by the contour cues and hence experiencing the usual Poggendorff effect. The illusion might result from spatial interaction between the transversals and the nearest edges of the contour cues, independent of whether $O$ perceives the subjective rectangle. Since the cause of the virtual-contour illusion was ambiguous, additional data were gathered that might allow one to choose between the above hypotheses.

\section{EXPERIMENT 2}

Method

Several additional figures were prepared in the manner described in Experiment 1. The $C$ and $T$ figures were drawn with 51-deg transversal tilts. Also, profile (P) figures were designed (at both transversal tilts) that consisted only of the transversal lines and black outline of the contour border closest to each transversal (see Fig. 1). The $P$ figures did not contain cues to suggest an enclosed rectangle.

Forty new paid $O$ s served in the experiment. The experimental procedures were identical to those of Experiment 1, except that each $O$ now made alignment judgments on the eight following stimuli: T-45, T-51, $\mathrm{C}-45, \mathrm{C}-51, \mathrm{P}-45, \mathrm{P}-51, \mathrm{~T}-45$, and T-51 deg. The additional presentation of the T-45- and T-51-deg stimuli constituted an imaginary Poggendorff condition, since the $\mathbf{S}$ was instructed to ". . imagine that two parallel lines are running vertically up the page so that the figure would look like this one [E shows $C$ figure]...." The two trials with the imaginary Poggendorff were always presented to $O$ last, the other six trials being presented in random order.

\section{Results}

Table 1 contains the mean amounts of illusion for each display and the associated standard errors of the mean. Two means, replications of Experiment 1, are not listed: C-45 deg, which produced values $(\bar{X}=6.68$, $S . E .=.60$ ) close to those found previously, and T-45 deg $(\bar{X}=.90$, S.E. $=.40$ ), which now gave a small but significant $(\alpha=.05)$ amount of illusion.

One of the main purposes of this experiment was to gather data on the profile display in order to compare it with data on the Farné figure from 
Experiment 1. A two-way analysis of variance was performed with type of figure $(F, P)$ as a between-Ss variable and transversal tilt (45 deg, $51 \mathrm{deg}$ ) as a within-Ss variable. Two effects were significant at the .01 level, the main effect of transversal tilt and the interaction between transversal tilt and figure type. (The main effect of type of figure was not significant, $F=1.25$.)

\section{DISCUSSION}

With the exception of the T-45-deg data from Experiment 2, the present results generally support the hypotheses of Weintraub and Krantz (1971) concerning the error-producing mechanisms in operation in the conventional Poggendorff display. Consequently, we will assume, temporarily, that the T-45-deg display gives zero illusion. Two error factors were presumed at work: (1) a powerful angle-misperception process which decreases as the acute angle formed between the parallel and the transversal increases, and (2) a tilt-assimilation factor toward horizontal. In the absence of objective parallels to form the critical angle, the angle-misperception factor is absent. At a transversal tilt of $45 \mathrm{deg}$, the tilt-assimilation factor is zero.

With a transversal tilt of $\mathbf{4 5} \mathrm{deg}$, the transversal-only and imaginary conditions have neither error factor operating, and the result is zero illusion. At $51 \mathrm{deg}$, there is assimilation of the transversal toward horizontal, which produces a small but significant illusion-the same amount for each display.

The conventional Poggendorff figure gives a large illusion at $45 \mathrm{deg}$ because of a powerful angle-misperception factor. At $51 \mathrm{deg}$, the presence of tilt assimilation cannot offset the decline in angle misperception; hence, the illusion decreases. The Weintraub-Krantz (1971) formula, $I=.162 \mathrm{~W} / \tan A$ (with $W=40 \mathrm{~mm}$ ), developed from similar experimental conditions, predicts a $6.48-\mathrm{mm}$ illusion for the 45-deg transversal tilt and a 5.26-mm illusion for the 51-deg tilt, both reasonably consistent with the new data.

Since neither the Farne figure nor the profile figure contain defined angles, they should behave quantitatively as well as qualitatively like the transversal-only figures. They do not. Both give a significant amount of illusion at a transversal tilt of $45 \mathrm{deg}$, and an even greater illusion at $51 \mathrm{deg}$. Additional factors may be operating in these displays. Note that both figures give increased illusions at the 51-deg transversal tilt, the reverse of the finding with the conventional Poggendorff display. Thus, whatever the nature of the error mechanisms operating in the $\mathbf{F}$ and $\mathbf{P}$ displays, they act in a qualitatively different manner from the angle-misperception factor responsible for the large illusion magnitudes with the $C$ displays.

Can we attribute a virtual-contour explanation to the Farne figure? The profile display induces an illusion with no cues to suggest subjective contours. The analysis of variance for $F$ and $P$ showed no significant main effect of figure type. There was, however, a significant interaction revealing that transversal tilt acted differentially upon the two figures. Thus the profile figure did not completely capture the essence of the Farné display. We must conclude that a virtual-contour Poggendorff of minimal magnitude does exist under certain viewing conditions.

Now to the discrepancy-the T-45-deg data from Experiment 2. Combining the T-45-deg data from both experiments leads to the conclusion that a small but significant illusion exists $(\bar{X}=.73 \mathrm{~mm}$, S.E. $=.28$ ). Thus, the 45-deg transversal-only figure seems to have a slight illusion-producing capability, independent of the two illusory mechanisms proposed above. If that conclusion is accepted, then the Farné, profile, and transversal-only figure displays act in a qualitatively similar fashion. The three figures differ, not in the direction of their effects, but in the illusion magnitudes produced by each. With only small quantitative values differentiating the displays, further analysis of whether different mechanisms are operating in the $F, P$, and $T$ stimuli seems premature. In any event, each of the three gives an increased illusion at the $51-\mathrm{deg}$ tilt, the opposite of what is found with the conventional Poggendorff. Hence, our main conclusion survives intact: the robust conventional Poggendorff display provides data which are both quantitatively and qualitatively different from that found with the $F$,
$P$, and $T$ displays.

In summary, the new evidence has added to our confidence that two factors, angle misperception and tilt assimilation, are major contributors toward a Poggendorff effect. Moreover, the actual presence of the parallels is needed for the operation of the first factor, which seems to be the stronger. While we must conclude that the virtual-contour Poggendorff display has limited illusory capabilities, our considered opinion is that this parallel-less Poggendorff will require a separate explanation because of its small magnitude and the fact that changing transversal tilt alters the illusion in a direction opposite to that of the conventional Poggendorff display. In other words, Farné has presented a Poggendorff of a different stripe.

\section{REFERENCES}

BLAKEMORE, C., CARPENTER, R. H. S. \& GEORGESON, M. A. Lateral inhibition between orientation detectors in the human visual system. Nature, 1970, 228, 37-39.

BURMESTER, E. Beitrag zur experimentellen Bestimmung geometrisch-optischer Täuschungen. Zeitschrift für Psychologie, 1896, 12, 355-394.

BURNS, B. D., \& PRITCHARD, R. Geometrical illusions and the response of neurons in the cat's visual cortex to angle patterns. Journal of Physiology, 1971. $213,599-616$.

CHIANG, C. A new theory to explain geometrical illusions produced by crossing lines. Perception \& Psychophysics, 1968, 3, 174-176.

CUMMING, G. D. A criticism of the diffraction theory of some geometrical illusions. Perception \& Psychophysics, 1968,4 , 375-376.

FARNé, M. On the Poggendorff illusion: A note to Cumming's criticism of Chung Chiang's theory. Perception \& Psychophysics, 1970, 8, 112.

GREEN, R. T. \& HOYLE, E. M. The influence of spatial orientation on the Poggendorff illusion. Acta Psychologica, 1964, 22, 348-366.

RESTLE, F. mlusions of bent line. Perception \& Psychophysics, 1969, 5, 273-274.

WEINTRAUB, D. J., \& KRANTZ, D. H. The Poggendorff illusion: Amputations, rotations, and other perturbations. Perception \& Psychophysics, 1971, 10, 25 7-264.

WEINTRAUB, D. J., \& VIRSU, V. The misperception of angles: Estimating the vertex of converging line segments. Perception \& Psychophysics, 1961, 9. 5-8.

WUNDT, W. Grundzüge der physiologischen Prychologie. Leipzig: Engelmann, 1893.

(Received for publication July 12, 1971.) 